\title{
Constraints from the Hubble Deep Field on High\# Redshift Quasar Models
}

\section{Citation}

Haiman, Zoltan, Piero Madau, and Abraham Loeb. 1999. "Constraints from the Hubble Deep Field on High\#Redshift Quasar Models." The Astrophysical Journal 514 (2): 535-43. https:// doi.org/10.1086/306969.

\section{Permanent link}

http://nrs.harvard.edu/urn-3:HUL.InstRepos:41393237

\section{Terms of Use}

This article was downloaded from Harvard University's DASH repository, and is made available under the terms and conditions applicable to Other Posted Material, as set forth at http:// nrs.harvard.edu/urn-3:HUL.InstRepos:dash.current.terms-of-use\#LAA

\section{Share Your Story}

The Harvard community has made this article openly available.

Please share how this access benefits you. Submit a story.

Accessibility 


\title{
CONSTRAINTS FROM THE HUBBLE DEEP FIELD ON HIGH-REDSHIFT QUASAR MODELS
}

\author{
Zoltán Haiman, ${ }^{1}$ Piero Madau, ${ }^{2}$ and Abraham Loeb ${ }^{1}$ \\ Received 1998 May 19; accepted 1998 November 3
}

\begin{abstract}
High-resolution, deep-imaging surveys are instrumental in setting constraints on semianalytical structure formation models in cold dark matter (CDM) cosmologies. We show here that the lack of unresolved $B$-band "dropouts" with $V>25$ mag in the Hubble Deep Field appears to be inconsistent with the number of "miniquasars" expected if massive black holes form with a constant universal efficiency in all CDM halos. To reconcile the models with the data, a mechanism is needed that suppresses the formation of active nuclei in halos with circular velocities $v_{\text {circ }} \lesssim 50-75 \mathrm{~km} \mathrm{~s}^{-1}$. This feedback naturally arises because of the photoionization heating of the gas by the UV background. We consider several alternative effects that would help reducing the number of miniquasars and find that these cannot alone account for the observed lack of detections. If reddening by dust can be neglected at early epochs, consistency with the optical data also requires that the luminous extent of dwarf galaxies at high redshifts be larger than a few percent of the virial radii of their dark matter halos, in order not to overpredict the number of pointlike $B$-band dropouts. Future deep observations in the $J$ and $H$ bands with NICMOS might reveal several $z \gtrsim 5$ objects per field or provide even stronger constraints on the models than existing $B, V$, and $I$ data.
\end{abstract}

Subject headings: cosmology: theory — galaxies: formation — quasars: general

\section{INTRODUCTION}

One of the primary goals of models for structure formation in the universe is to explore the formation process of luminous objects such as quasars or galaxies and to explain the shape and evolution of their observed luminosity functions (LFs). Recent theoretical work has focused on the formation of galaxies within the framework of hierarchical structure formation in cold dark matter (CDM) cosmologies, employing either three-dimensional numerical simulations (Gnedin \& Ostriker 1997; Zheng et al. 1997) or semianalytic modeling (Kauffman, Guiderdoni, \& White 1994; Cole et al. 1994; Baugh et al. 1998; Somerville \& Primack 1998; Haiman \& Loeb 1997, hereafter HL97). These calculations have been quite successful in reproducing the observed Tully-Fisher relation in the local universe, the faint galaxy LFs out to moderately high redshifts $z \lesssim 3$, and the recently discovered clustering of Lymanbreak galaxies at $z \sim 3$ (Baugh et al. 1998; Governato et al. 1998; Steidel et al. 1998). Hierarchical models were also applied to the more complex problem of quasar formation (Efstathiou \& Rees 1988; Haehnelt \& Rees 1993; Small \& Blandford 1992) and were shown to reproduce the observed evolution in the quasar LF at redshifts $z \gtrsim 2$ under a minimal set of simplifying assumptions (Haiman \& Loeb 1998b, hereafter HL98; see also Haehnelt, Natarajan, \& Rees 1998).

The above models were not tested in detail at redshifts much higher than $z \sim 3$ because of the lack of related observational data. Such tests are desirable from the theoretical point of view, since the model predictions are expected to be more robust at higher redshifts, when the stars and quasars first condensed out of the well-defined set of initial conditions in the early universe. The exponentially low abun-

\footnotetext{
${ }^{1}$ Astronomy Department, Harvard University, 60 Garden Street, Cambridge, MA 02138.

${ }^{2}$ Space Telescope Science Institute, 3700 San Martin Drive, Baltimore, MD 21218.
}

dance of collapsed objects at very early times reduces the probability for complicated merger events. Moreover, the chemical and thermal conditions inside objects that assembled out of the pristine intergalactic gas are better defined before metal enrichment and thermal feedback from multiple generations of stars started to affect the appearance of quasars and galaxies. To date, the deepest optical image of the universe is the Hubble Deep Field (HDF; Williams et al. 1996), which surveyed a 2.3 $\times 2.3$ field in four bandpasses down to $\sim 29$ mag. Although this image has revealed a large number of galaxies of different morphological types, a surprisingly large fraction of the sky was found to remain dark at the HDF detection limit (e.g., Vogeley 1998). The Next Generation Space Telescope (NGST), scheduled for launch in 2007, is planned to have a sensitivity of $1 \mathrm{nJy}$ in the 1-3.5 $\mu \mathrm{m}$ range and should be able to detect globular cluster scale objects out to $z \sim 10$ (see Smith \& Koratkar 1998).

The properties of faint extended sources in the HDF (Madau et al. 1996) have been well described by detailed semianalytic galaxy formation models (Baugh et al. 1998). On the other hand, the HDF has revealed only a handful of faint unresolved sources (Méndez et al. 1996) and none with the colors expected for high-redshift objects. The purpose of the present paper is to show that the lack of pointlike sources in the HDF can be used to constrain models for the formation of cosmic structure at redshifts higher than previously possible $(z \gtrsim 4)$. Although the theoretical models described here suffer from large uncertainties and should only be considered as illustrative, they are only used to make relatively mild extrapolations from existing optical data.

The rest of this paper is organized as follows. The observational data and color-selection criteria are summarized in $\S 2$ and $\S 3$, and the simplest versions of the semianalytic CDM models are outlined in $\S 4$. In $\S 5$ we compute the optical and infrared quasar number counts in these models, and show that the predictions from standard scenarios without a UV feedback appear to be inconsistent with the 


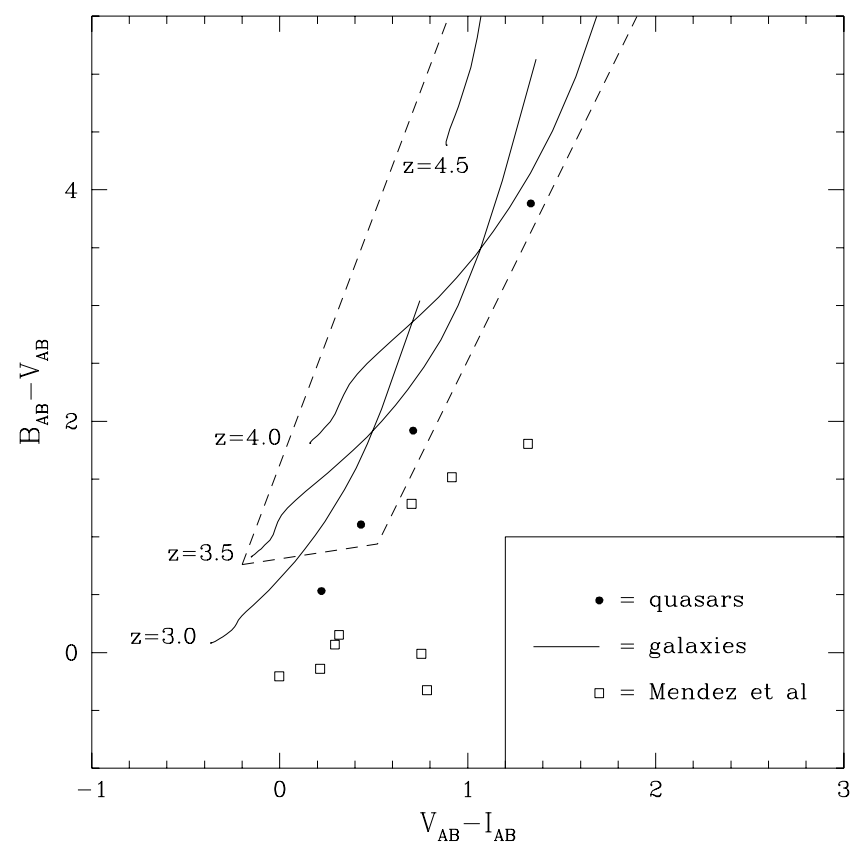

FIG. 1.-Theoretical $B-V$ vs. $V-I$ color-color diagram for $z>3$ objects. The solid dots show quasars whose intrinsic colors are assumed not to change with cosmic time, while the solid lines show galaxies with ages of $0-10^{9} \mathrm{yr}$. The data points (open squares) are the nine faint $(V>25)$, unresolved objects from Méndez et al. (1996). The trapezoidal region enclosed by the dashed lines should selects all unreddened sources with $z \gtrsim 3.5$.

observed lack of high- $z$ sources. We also show that models with a UV feedback are marginally consistent with the data. In $\S 6$ we describe possible modifications of these models that could reduce the predicted counts. In $\S 7$ we discuss the expected galaxy number counts. In $\S 8$ we present our conclusions and argue that future IR observations in the $J$ and

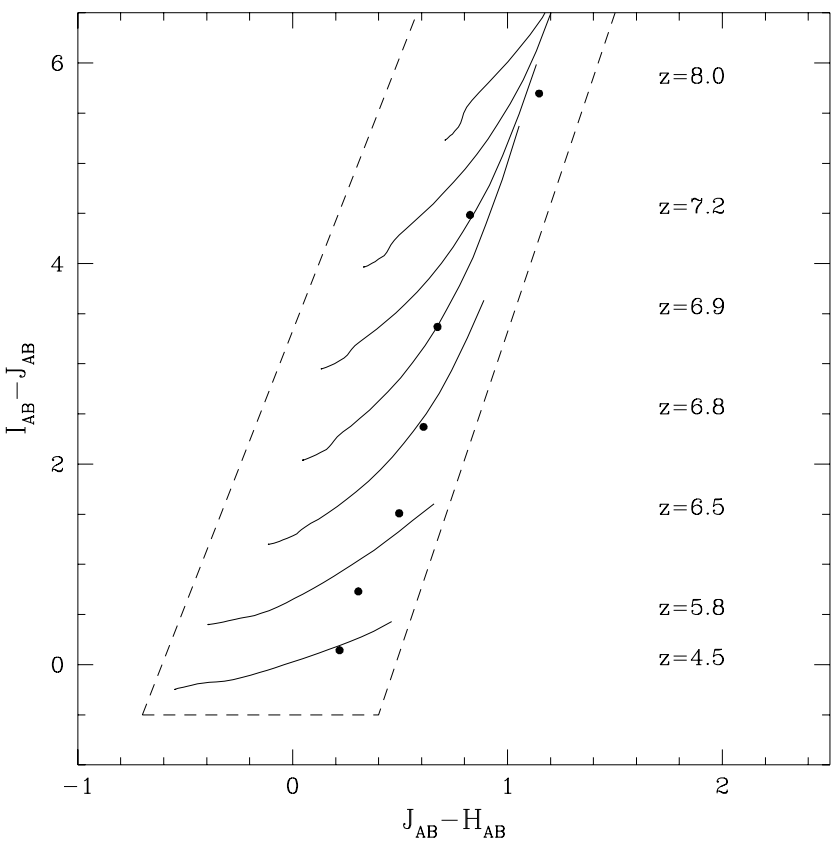

FIG. 2.-Theoretical $I-J$ vs. $J-H$ color-color diagram for $4.5 \lesssim z \lesssim 8$ objects. The solid dots show the expected colors of quasars, while the solid lines show galaxies with ages $0-10^{9}$ yr. The trapezoidal region selects objects with $z \gtrsim 4.5$.
$H$ bands with NICMOS may in principle be used to place even stronger constraints on this type of scenarios.

\section{HIGH-REDSHIFT QUASAR CANDIDATES IN THE HDF}

Several surveys of unresolved objects in the HDF have appeared in the literature (Elson, Santiago, \& Gilmore 1996; Flynn, Gould, \& Bahcall 1996; Méndez et al. 1996). Méndez et al. (1996) were able to identify 14 pointlike objects, with only six of them at magnitudes fainter than $V=26.5 .^{3}$ The faintest sources in their sample tend to have rather blue $B-V$ and $V-I$ colors (see also Elson et al. 1996). These authors point out that although the stargalaxy separation software fails above $V \approx 27.5$, misclassification at fainter magnitudes should turn more galaxies into stars than vice versa, as the overwhelming majority of objects in the HDF are galaxies, and diffuse and faint features disappear first. Hence the number of pointlike sources detected down to $V=29$ should be considered as an absolute upper limit to the number of stars or quasars observed in this magnitude interval. In analogy with star-forming galaxies, high-redshift $(z \gtrsim 3.5)$ quasars are expected to have very red $B-V$ and reddish $V-I$ colors because of continuum absorption and line blanketing from neutral hydrogen along the line of sight. As was pointed out by Elson et al. (1996), however, none of the stellar objects in the HDF has the colors expected for high- $z$ quasars. A recent detailed search for faint quasars in the HDF by Conti et al. (1998) confirms the lack of a population of very red compact sources; no $z>3.5$ quasar candidates were found down to a $50 \%$ completeness limit at $V=29.6$.

\section{IDENTIFYING SOURCES AT $z \gtrsim 3.5$}

Before comparing our models directly with the observations, we show in Figure 1 the location of the nine faint $V>25$ sources detected by Méndez et al. (1996) on a $B-V$ versus $V-I$ color-color diagram (open squares). The solid lines in this figure show the predicted trajectories of model galaxies born at redshifts $3<z<4.5$ and passively evolving for $10^{9} \mathrm{yr}$ after an instantaneous burst of star formation. The filled dots show the expected colors of quasars at the same redshifts. Unlike galaxies, which change their colors because of the evolution of their stellar population, we have assumed that the aging of quasars only changes the overall normalization, not the shape of their intrinsic spectra (see $\S 4$ below for details of our model). While all $z>3.5$ objects are expected to fall within the trapezoidal region shown in the figure, none of the Méndez et al. (1996) sources appears to have the colors predicted for a QSO or a galaxy at high- $z$. One caveat to the color-selection method is that some QSOs could have unusual colors. Indeed, the three sources that have $(B-V)>1$ in the Méndez sample are found close to the border of the QSO selection region. However, all of these objects are rather bright $(V=25,25.5$, 26.3), and if they are indeed QSOs, they are likely to have low $(z<3)$ redshifts. We note that if these three objects turned out to be QSOs at $z>3.5$, then our models would underpredict the number of bright $(V \sim 26)$ quasars, in addition to overpredicting the faint counts (see Fig. 3 and discussion below).

\footnotetext{
${ }^{3}$ Magnitudes throughout this paper are in the $\mathrm{AB}$ system, i.e., $m_{\mathrm{X}, \mathrm{AB}}=$ $-2.5 \log \left[f_{\mathrm{X}} / \mathrm{ergs} \mathrm{s}^{-1} \mathrm{~cm}^{-2} \mathrm{~Hz}^{-1}\right]-48.6$.
} 
We note that it should be possible to identify objects at $z>4.5$ in much the same way as the $z \sim 4$ galaxies are identified by the $B$ dropout technique. Since at higher redshifts line blanketing and Lyman-continuum absorption are even more pronounced because of the larger number of intervening systems along the line of sight, a straightforward extension of the dropout technique, using $I, J$, and $H$ data, should work fairly well. In analogy with the $z \gtrsim 3.5 B$ dropout galaxies, we expect $V, I$, and $J$ dropout sources to flag the redshifts $z \gtrsim 5,7$, and 9. The $B-V$ versus $V-I$ diagram used to identify $z \sim 4$ star-forming objects (Madau et al. 1996) could then be replaced by a $I-J$ versus $J-H$ color-color diagram as a probe of the redshift range $4.5<z<8$, as is illustrated in Figure 2. Unreddened galaxies and quasars at $z>4.5$ are expected to be confined to the trapezoidal region indicated in this figure, with negligible contamination from foreground stars.

\section{MODEL DESCRIPTION}

In modeling the abundance of high-redshift sources, we assume that the formation of dark matter halos follows the Press-Schechter (1974) theory. Hence the net rate of change in the comoving number density of halos with mass $M_{\text {halo }}$ is given by the derivative $d / d z\left(d n_{\mathrm{c}} / d M\right)$, where $d n_{\mathrm{c}} / d M$ is the Press-Schechter mass function (comoving number density per unit mass). This rate includes a negative contribution from the disappearance of small halos in merger events; the net $d / d z\left(d n_{\mathrm{c}} / d M\right)$ becomes negative when mergers dominate, i.e., for masses below a characteristic mass scale $M_{*}(z)$ at any given redshift $z$. Quasars are short-lived, and we assume that their formation rate is proportional to the formation rate of new halos. The negative contribution to this rate from merger events should therefore be ignored. For relatively bright quasars at high redshifts (and for all cases in our standard $\Lambda$ CDM model), the Press-Schechter formation rate is adequate, since the typical halo mass $M_{\text {halo }}$ is above $M_{*}(z)$. Otherwise, we make the conservative assumption that no quasars form in halos with a mass $M_{\text {halo }}<$ $M_{*}(z)$ and therefore set $d / d z\left(d n_{\mathrm{c}} / d M\right)=0$ (see discussion below).

After each halo collapses and virializes, its gas might condense into stars or a quasar black hole, provided it can cool efficiently from its initial virial temperature. Efficient cooling via collisional excitation of atomic hydrogen requires the halo mass to be at least $M_{\min } \sim 10^{8} M_{\odot}[(1+$ $z) / 11]^{-3 / 2}$ (Haiman et al. 1997). In halos that exceed this mass, a fraction $\epsilon_{\star}$ of the gas might fragment into stars, forming a dwarf galaxy with a stellar mass of $M_{\text {star }}=$ $\epsilon_{\star} M_{\text {halo }}$. A different fraction $\epsilon_{Q}$ of the gas might accrete onto a massive central black hole with a mass $M_{\mathrm{bh}}=$ $\epsilon_{Q} M_{\text {halo }}$ and exhibit a low-luminosity quasar activity (Eisenstein \& Loeb 1995; Loeb 1998). The efficiencies $\epsilon_{\star}$ and $\epsilon_{Q}$ depend on several parameters, such as the redshift, halo mass, or the initial angular momentum of the gas. However, the simplest assumption to make is that the efficiencies are the same for all halos.

An important effect we need to consider is an external feedback from photoionization, which exerts its influence by heating the gas before it is able to cool and condense inside the dark matter potential wells. Such a feedback is an inevitable consequence of the background UV flux that builds up after the reionization epoch, when the cosmological $\mathrm{H}$ II regions have overlapped and the universe is transparent to the ionizing flux from each individual source.
Several authors have discussed the consequences of this feedback (Efstathiou 1992; Quinn, Katz, \& Efstathiou 1996; Thoul \& Weinberg 1996; Navarro \& Steinmetz 1997). According to the spherically symmetric simulations of Thoul \& Weinberg (1996), a UV background at the level expected from the proximity effect $\left(J_{21}=0.1-1\right.$; Bajtlik, Duncan, \& Ostriker 1988) completely suppresses the cooling and collapse of gas inside halos with circular velocities $v_{\text {circ }} \lesssim 35 \mathrm{~km} \mathrm{~s}^{-1}$ and substantially reduces (by $\approx 50 \%$ ) the mass of cooled baryons in systems with $v_{\text {circ }} \lesssim 50 \mathrm{~km}$ $\mathrm{s}^{-1}$. While this type of feedback has been mainly invoked to suppress the formation of dwarf galaxies (Ikeuchi 1986; Efstathiou 1992; Babul \& Rees 1992), it is also natural to expect that a similar mechanism may inhibit the formation or fueling of quasar black holes in shallow potential wells. In our standard model we therefore impose a minimum circular velocity of $v_{\text {circ }}=50 \mathrm{~km} \mathrm{~s}^{-1}$ for the halos of both luminous quasars and galaxies that form after the reionization epoch. This constraint results in a minimum halo mass of $M_{\min } \sim 3 \times 10^{9} M_{\odot}[(1+z) / 11]^{-3 / 2}$, which is 2 orders of magnitude higher than the cutoff mass obtained from the cooling argument.

The value of $\epsilon_{\star}$ can be derived from the requirement that the resulting stellar population reproduces the metallicity observed at $z \sim 3$ in the Ly $\alpha$ forest, $10^{-2} \lesssim Z_{\text {Ly } \alpha} \lesssim 10^{-3}$ $Z_{\odot}$ (Tytler et al. 1995; Songaila \& Cowie 1996; Songaila 1997). Assuming a Scalo (1986) stellar initial mass function (IMF) and using carbon as a measure of metallicity, this yields $0.017 \lesssim \epsilon_{\star} \lesssim 0.17$ (HL97). Similarly, the value of $\epsilon_{Q}$ for high-redshift quasars can be found by matching the observed LF of quasars at intermediate redshifts, $\phi(L, z)$. Assuming that all quasars shine with a universal light curve in Eddington units [i.e., that $L(t)$ scales linearly with the black-hole mass, $\left.L(t)=M_{\text {bh }} f(t)=\epsilon_{Q} M_{\text {halo }} f(t)\right]$, the constant $\epsilon_{Q}$ and the function $f(t)$ can be adjusted so as to produce the observed $\phi(L, z)$ at $2.6 \lesssim z \lesssim 4$ based on the relation

$$
\begin{aligned}
\phi(L, z)= & \int_{z}^{\infty} \int_{M_{\min }}^{\infty} d M_{\text {halo }} d z^{\prime} \\
& \times \frac{d^{2} n_{\text {halo }}}{d M_{\text {halo }} d z^{\prime}} \delta\left[L-\epsilon_{Q} M_{\text {halo }} f\left(t_{z, z^{\prime}}\right)\right],
\end{aligned}
$$

where $t_{z, z^{\prime}}$ is the time elapsed between the redshifts $z$ and $z^{\prime}$. To account for the decline of the observed quasar population at redshifts below $z \sim 2.6$ would require additional modeling but should not affect our conclusions here. The fit to the observational data for $\phi(L, z)$ (in the rest frame $B$ band) given by Pei (1995) yields $\epsilon_{Q} \sim 6 \times 10^{-4}$ and a lightcurve shape that is well approximated by an exponential, $f(t) \sim \exp \left(-t / t_{0}\right)$, with $t_{0}=10^{5.8} \mathrm{yr}$ in a flat $\Lambda$ CDM cosmology with $\Omega_{\Lambda}=0.65$ and a Hubble constant of $h=0.65$ (HL98). Although the best-fit values for $t_{0}$ and $\epsilon_{Q}$ vary with cosmology (see discussion in $\S 6$ ), the adopted procedure fixes $\epsilon_{Q}$ and $t_{0}$ to within a factor of 2 in any given cosmological model (HL98). We note that $t_{0}$ could be increased substantially if black holes formed in only a fraction $f$ of all halos. For example, Eisenstein \& Loeb (1995) have argued that only the small subset of initial seed perturbations with sufficiently slow initial rotation can form black holes. In order to recover the fit to the LF, the lifetime would then need to be increased to $\sim t_{0} / f$.

The Press-Schechter halo formation rate depends on the cosmology and power spectrum. For our "standard" 
scenario we have adopted the concordance model of Ostriker \& Steinhardt (1995), i.e., a flat $\Lambda$ CDM model with a slightly tilted power spectrum $\left(\Omega_{0}, \Omega_{\Lambda}, \Omega_{\mathrm{b}}, h, \sigma_{8 h^{-1}}, n\right)=$ $(0.35,0.65,0.04,0.65,0.87,0.96)$. Convenient expressions for the differential volume element, luminosity distance, and time-redshift relation in this model were given in terms of elliptic integrals by Eisenstein (1997); an accurate fitting formula for the growth function is given by Carroll, Press, \& Turner (1992). We also consider two open models without a cosmological constant, one with $\Omega_{0}=0.35$ and the other with $\Omega_{0}=0.2$. In order to remain consistent with the power-spectrum normalization on both $C O B E$ and cluster scales (Viana \& Liddle 1996), we change the spectral index to $n=1.15$ and 1.2, respectively, in these models.

For our quasar template spectrum, we have used the average spectrum derived from the observations of 47 quasars by Elvis et al. (1994). The stellar template spectra have been computed from the low-metallicity $(Z=$ $4 \times 10^{-4}$ ) population synthesis models of Bruzual \& Charlot (1996), assuming an instantaneous burst of star formation and a Scalo (1986) IMF. Note that a Salpeter IMF produces about twice as many UV photons as the Scalo IMF for the same level of carbon enrichment of the intergalactic medium (IGM). We find that adopting a Salpeter IMF would increase our predicted number counts down to $V \sim 30$. In our standard model, the increase is negligible at $V=30$ but is an order of magnitude higher at $V=22$.

\section{NUMBER COUNTS}

In comparing our models with the observations, it is useful to clarify our naming conventions. Observationally, only bright $\left(M_{B} \lesssim-23\right)$ objects are usually termed QSOs, while fainter objects are referred to as active galactic nuclei. The faintest sources addressed below $(V \sim 29$ at $z \sim 4)$ have, in our assumed cosmology, an absolute magnitude of $M_{\mathrm{UV}} \sim-19$ and, if nearby, would be classified as Seyfert galaxies. To make explicit the fact that the constraints below arise from the very faint end of the quasar LF, we will hereafter refer to accreting black holes with $M_{\mathrm{UV}} \gtrsim-23$ as "miniquasars."

In both the quasar and galaxy models, the number of sources located between redshifts $z_{\min }$ and $z_{\max }$ per unit solid angle, with observed visible magnitude between $V$ and $V+d V$, is given by

$$
\frac{d N}{d \Omega d V}\left(V, z_{\min }, z_{\max }\right)=\int_{z_{\min }}^{z_{\max }} d z\left(\frac{d V_{\mathrm{c}}}{d z d \Omega}\right) n_{\mathrm{c}}(z, V),
$$

where $d V_{\mathrm{c}} / d z d \Omega$ is the comoving volume element per unit redshift per unit solid angle (Eisenstein 1997), and $n_{\mathrm{c}}(z, V)=d N / d Z d V$ is the comoving number density of objects at a redshift $z$ with observed magnitudes between $V$ and $V+d V$.

Based on the halo formation rate $d^{2} n / d M d z, n_{c}$ is given by a sum over halos of different ages that exist at each redshift,

$$
n_{\mathrm{c}}(z, V)=\int_{z}^{\infty} d z^{\prime} \frac{d M_{\text {halo }}}{d V}\left(z, z^{\prime}, V\right)\left|\frac{d^{2} n_{\mathrm{c}}}{d M d z^{\prime}}\right|_{M_{\text {halo }}\left(z, z^{\prime}, V\right), z^{\prime}},
$$

where the factor $d M_{\text {halo }} / d V$ converts the number density per unit mass interval to number density per unit flux interval.
The $V$ magnitude in the $\mathrm{AB}$ system is given by

$$
V=-2.5 \log f_{V}-48.60,
$$

where the observed $V$-band flux $f_{V}\left(z, z^{\prime}, M_{\text {halo }}\right)$ from a halo at redshift $z$ with a mass $M_{\text {halo }}$ that has formed at $z^{\prime} \geq z$ equals (in units of ergs s ${ }^{-1} \mathrm{~Hz}^{-1} \mathrm{~cm}^{-2}$ ),

$$
\begin{aligned}
f_{V}\left(z, z^{\prime},\right. & \left.M_{\text {halo }}\right)=\frac{(1+z) \epsilon M_{\text {halo }}}{4 \pi d_{L}(z)^{2}} \\
\times & {\left[\int_{0}^{\infty} d v T_{V}(v) \exp [-\tau(v, z)] j\left(v(1+z), t_{z, z^{\prime}}\right)\right] } \\
\times & {\left[\int_{0}^{\infty} d v T_{V}(v)\right]^{-1} . }
\end{aligned}
$$

Here $d_{L}(z)$ is the luminosity distance; $\epsilon=\epsilon_{Q} \equiv 6 \times 10^{-4}$ or $\epsilon=\epsilon_{\star} \equiv\left(\Omega_{\mathrm{b}} / \Omega_{0}\right) \times(0.017-0.17)=0.002-0.02$ is the fraction of halo mass in the form of the central black hole or stars, respectively; $\tau(v, z)$ is the effective optical depth associated with intervening Ly $\alpha$ forest clouds and Lyman-limit systems between redshifts 0 and $z$ at the observed frequency $v$ (Madau 1995); $T_{V}(v)$ is the frequency-dependent transmission of the $V_{606}$ filter; ${ }^{4}$ and $j\left(v, t_{z, z^{\prime}}\right)$ is the template luminosity per unit (stellar or black hole) mass for either stars or quasars, in units of ergs s${ }^{-1} \mathrm{~Hz}^{-1} M_{\odot}^{-1}$. The time dependence of $j(v)$ is obtained from our fitted light-curve function $L(t)$ for quasars and from tabulated evolutionary tracks for stars (Schaller et al. 1992; see HL97 for details). We suppressed the emergent flux from all objects at emission frequencies above Ly $\alpha$ before reionization occurs (assuming sudden reionization at the redshift $z=13$ or 9 for stars and $z=11.5$ for quasars; see HL98 for details), since the GunnPeterson optical depth of $\mathrm{H} \mathrm{I}$ at these frequencies is exceedingly high prior to reionization.

The expected counts of $z>3.5$ quasars in the $V$ band are shown by the solid lines, labeled A, in Figure 3. The bottom panel also shows the observational data points from the $B$ band (Pei 1995), converted to $V$ counts using the shape of our template spectrum. By construction, the differential model number counts for quasars in the narrow redshift interval $3<z<3.2$ are consistent with the data between $17<V<22.5$. The upper panel in this figure shows the cumulative counts of $z>3.5$ QSOs and reveals that down to the $V=29$ limit of the HDF, our standard model predicts about four such miniquasars. Since no unresolved sources at $z>3.5$ have been found in the HDF, a model that is consistent with the HDF data at the $2 \sigma$ level should predict no more than two such high- $z$ miniquasars. On the other hand, since the observations are between $90 \%$ and $50 \%$ complete down to $V=29$ (Conti et al. 1998), a prediction of four miniquasars would still be allowed. Our standard miniquasar scenario therefore appears to be marginally consistent with the HDF data. Although the existing data on the LF only constrains the individual quasar model parameters such as $\epsilon_{Q}$ and $t_{0}$ to within a factor of $\sim 2$, the $z>3.5$ number counts are predicted to a much better accuracy, since the model is calibrated by simultaneously adjusting $\epsilon_{Q}$ and $t_{0}$ to reproduce the quasar LF at $z \sim 3$. As a result, an overprediction of the miniquasar counts by a

\footnotetext{
${ }^{4}$ Available at http://www.stsci.edu/ftp/instrument_news/WFPC2/ Wfpc2_faq/wfpc2_phot_faq.html.
} 


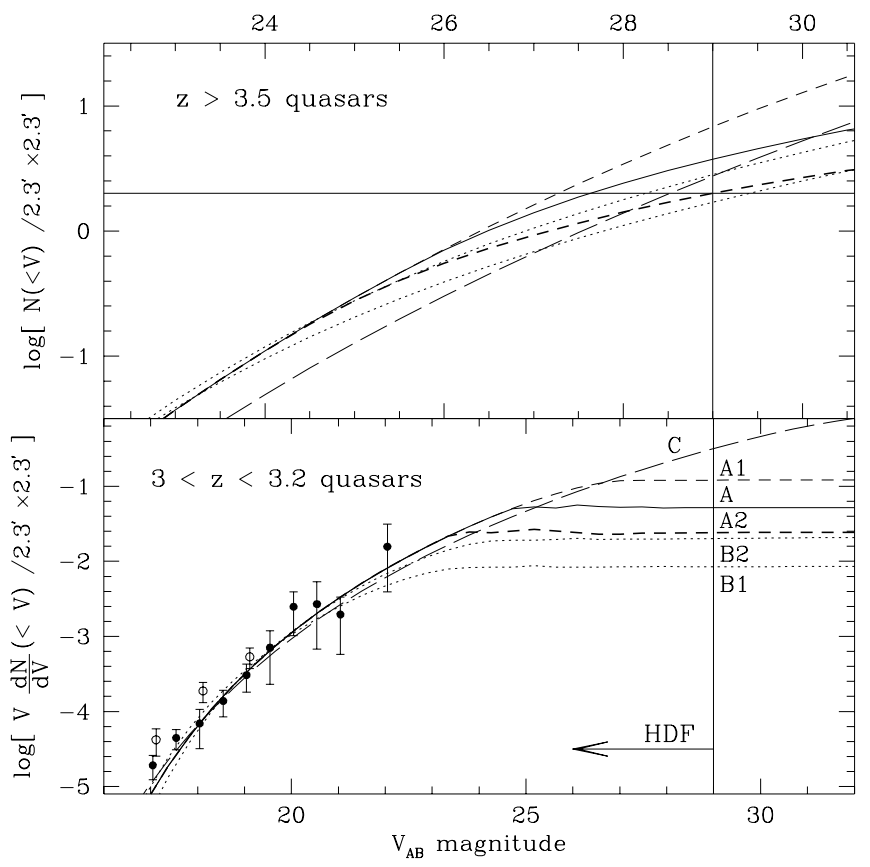

Fig. 3. $-V$-band number counts for high-redshift quasars. The lower panel shows differential counts for $3<z<3.2$, as well as data adapted from Pei (1995). The upper panel shows cumulative number counts for $z>3.5$. The curves labeled A show predictions of our standard $\Lambda$ CDM model with $M_{\mathrm{bh}} \propto M_{\text {halo }}$. The curves labeled A1 show the counts in a model with no UV feedback. The curves labeled A2 correspond to a model with halo circular velocities $v_{\text {circ }} \geq 75 \mathrm{~km} \mathrm{~s}^{-1}$. The curves labeled $\mathrm{B} 1$ and B2 correspond to open CDM models with $\Omega_{0}=0.35$ and 0.2 , respectively, and the curves labeled $\mathrm{C}$ corresponds to a "tilted" model with $M_{\mathrm{bh}} \propto$ $M_{\text {halo }}^{1.5}$.

factor of 2 could still be significant. It is important to remember here that in our standard model we have already eliminated miniquasars in halos with $v_{\text {circ }}<50 \mathrm{~km} \mathrm{~s}^{-1}$ in order to mimic the effect of photoionization heating. The thin dashed curves in Figure 3, labeled A1, show the number counts in a model without this UV feedback and reveal a prediction of $\sim 8$ objects to $V=29$. The overprediction in this model would be rather severe, a factor of $\sim 4$.

Finally, it is worthwhile to compare the miniquasar LF predicted here with the ad hoc fitting formulae derived by Pei (1995). While these formulae are not based on any underlying physics, they provide a useful analytic reference. Although our model agrees well with Pei's double powerlaw expression in the magnitude range where the data are available, it predicts $\sim 10$ times more miniquasars at the redshifts $(z>3.5)$ and magnitudes $\left(V \sim 29\right.$ or $\left.L_{B, \odot} \sim 10^{9.5}\right)$ relevant here (see HL98). In fact, the faint end slope of Pei's fitting function could be as steep as $\beta \approx 1.8$ (compared with $\beta=1.64$ in Table 1 of that reference) and still be consistent with the HDF constraints $(\leq 2$ miniquasars at high redshifts).

The expected number counts of $z>3.5$ galaxies in our standard model are shown by the curves labeled A in Figure 4. The solid and dashed lines bracket the expected metallicity range of the IGM at $z \approx 3,10^{-3} Z_{\odot}<Z<10^{-2} Z_{\odot}$. Depending on the metallicity calibration, the total number of galaxies observable down to $V=29$ mag is between 20 and 140. This exceeds the predicted miniquasar counts by a factor of 5-35 and is 10-100 larger than what would be allowed for point sources in the HDF. However, depending

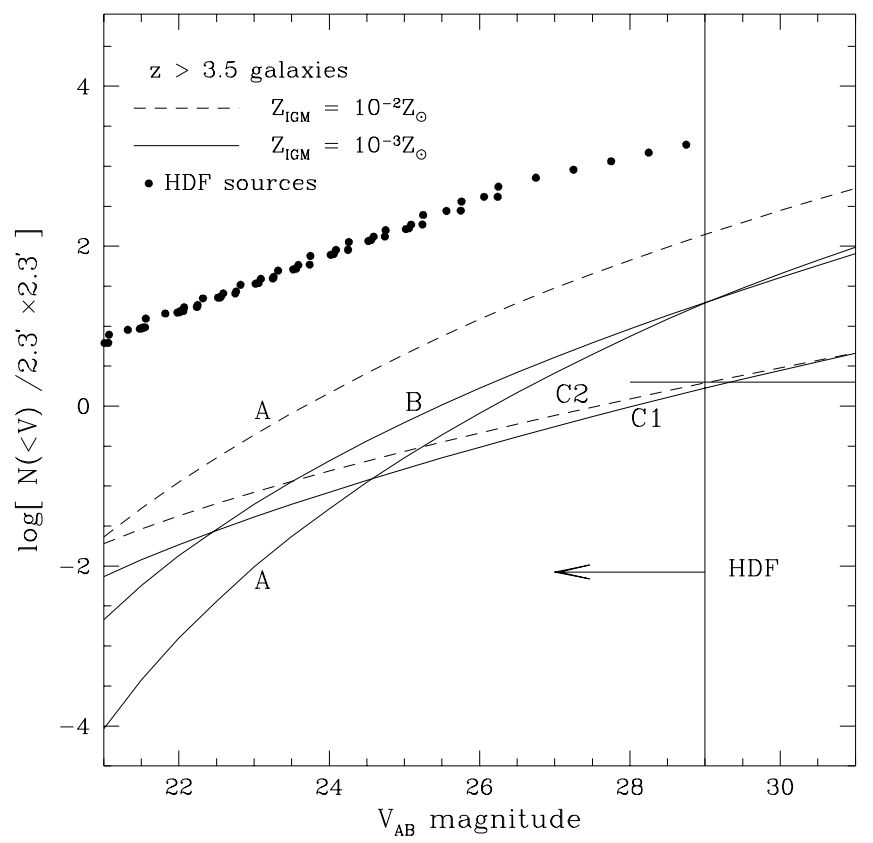

FIG. 4. $-V$ counts for high-redshift galaxies in the simplest model with $M_{\text {star }} \propto M_{\text {halo }}$. The solid lines correspond to low star formation efficiencies $\left(M_{\text {star }}=0.017 M_{\text {halo }}\right)$ that produce an IGM metallicity of $10^{-3} Z_{\odot}$, and the dashed lines correspond to a star formation efficiency that is 10 times higher. The curves labeled A correspond to our standard model. The curve labeled B shows the counts in the open CDM model. The curves labeled $\mathrm{C} 1$ and $\mathrm{C} 2$ correspond to models with $M_{\text {star }} \propto M_{\text {halo }}^{3}$ (for $Z_{\mathrm{Ly} \alpha}=10^{-3} Z_{\odot}$ ) and $M_{\text {star }} \propto M_{\text {halo }}^{3.5}$ (for $Z_{\mathrm{Ly} \alpha}=10^{-2} Z_{\odot}$ ), respectively. The solid dots show all extended sources detected in the HDF in the $V$ band (Williams et al. 1996).

on their assumed intrinsic sizes, some or most of the $z>3.5$ galaxies could actually be resolved (see discussion below). We also note that recent revisions of the $\mathrm{C} / \mathrm{H}$ ratio in these absorbers prefer lower metallicities, closer to $10^{-3} Z_{\odot}$ (Songaila 1997).

\section{REDUCING THE $z>3.5$ MINIQUASAR COUNTS}

As the simplest scenarios with UV feedback appear to be only marginally consistent with the $V$ counts of faint quasars, the question naturally arises: how should one modify the models in order to reduce even further the expected number of faint pointlike objects? In general, the theoretical number counts are sensitive to the underlying cosmology and power spectrum, the relation between the halo mass and the emitted luminosity, and the time evolution of each object, including its merger history.

The simplest explanation for the observed lack of miniquasars would be a more effective UV feedback mechanism, resulting in a higher minimum halo size for luminous objects. In Figure 3, the lines labeled A2 show the quasar number counts in a model with $v_{\text {circ }}>75 \mathrm{~km} \mathrm{~s}^{-1}$. The top panel of this figure demonstrates that with this cutoff value of $v_{\text {circ }}$, the $z>3.5$ counts are reduced to two objects, i.e., consistent with the observed lack of detections. This required cutoff value is higher than the values obtained from spherical simulations (Thoul \& Weinberg 1996). With a UV background at the level of $0.3<J_{21}<1$, the cooled baryon fraction is fully suppressed for halos with $v_{\text {circ }} \lesssim 35$ $\mathrm{km} \mathrm{s}^{-1}$, suppressed by $50 \%$ for $v_{\text {circ }} \lesssim 50 \mathrm{~km} \mathrm{~s}^{-1}$, and unaffected for circular velocities above $v_{\text {circ }} \gtrsim 75 \mathrm{~km} \mathrm{~s}^{-1}$. However, using three-dimensional simulations, Navarro \& 
Steinmetz (1997) find the UV feedback to be more effective, and their circular velocity thresholds are $v_{\text {circ }}=80-200 \mathrm{~km}$ $\mathrm{s}^{-1}$, exceeding the required value $v_{\text {circ }}=75 \mathrm{~km} \mathrm{~s}^{-1}$ derived here.

In the absence of a photoionization feedback, another straightforward approach to reduce the faint counts is to make the slope of the LF shallower. If the luminosity of each object were steady and proportional to the halo mass, $L \propto M$, then the LF would have been related to the mass function simply by $\phi(L)=d n_{\mathrm{c}} / d M \times d M / d L \propto d n_{\mathrm{c}} / d M$, i.e., the LF would have had the same slope as the PressSchechter mass function. At low masses, $d n_{\mathrm{c}} / d M \propto$ $M^{-2+(n-1) / 6}$, independent of cosmology and only very weakly dependent on the power spectrum index $n$. By introducing a nonlinear scaling between luminosity and halo mass, $L \propto M^{1+\alpha}$ (see discussion below), one could tune the LF to have a shallower slope, $\phi(L) \propto L^{-1-1 /(1+\alpha)}$.

In reality, each source shines only for a limited amount of time $\Delta t$, which is much shorter than the Hubble time. This results in $\phi(L) \sim \Delta t \times d n_{\mathrm{c}} / d M d t \times d M / d L(t)$, where $d M / d L \propto \exp \left(-t / t_{0}\right)$ for our exponential light curve. Here a complication arises, because at masses below $M_{*}$, the time derivative of the mass function is negative, $d n_{\mathrm{c}} / d M d t<0$. Physically, this derivative is the sum of a positive term (the formation rate of new halos with mass $M$ ) and a negative term (the disappearance rate of existing halos due to mergers), and while the formation rate exceeds the merger rate for $M>M_{*}$, the latter dominates for $M<M_{*}$. The exact ratio between the formation and merger rates requires a scheme that goes beyond the Press-Schechter theory and describes the merging history of halos (Lacey \& Cole 1993; Kauffmann \& White 1993). For the sake of simplicity, we will assume here that no halos form below the threshold mass $M_{*}$ and that no halos are destroyed by mergers above this mass, i.e., we simply set the derivative $d n_{\mathrm{c}} / d M d t$ to zero for $M<M_{*}$ and to the Press-Schechter value for $M>M_{*}$. Although this assumption is ad hoc, it can only underestimate the number of objects. Therefore, the reduction in the number counts derived here should be considered as the minimum amount of change that is necessary in order to fit the observations.

Under the above prescription, the LF flattens to $\phi(L) \propto 1 / L$ at luminosities corresponding to $M<M_{*}$. The cosmological parameters $\left(\Omega_{0}, \Lambda, H_{0}\right)$ and the power spectrum parameters $\left(\sigma_{8 h^{-1}}, n\right)$ flatten the number counts at $V=29$ (with $V d N / d V=$ const) in models where low-mass halos form at sufficiently high redshift. Successful models raise the threshold mass $M_{*}$ at $3 \lesssim z \lesssim 6$ to high enough values that $V \sim 29$ objects become associated with sub- $M_{*}$ halos. For example, at $z \sim 3$ the halo mass corresponding to $V \sim 29$ in our models is $\sim 10^{8-9} M_{\odot}$. In general, $M_{*}$ is high in models with large values of $\sigma_{8 h^{-1}}, n$, and $\Omega_{0}$ and low values of $\Lambda$. These parameters are related by the normalization of the power spectrum on the COBE (X-ray cluster) scale, which requires $\sigma_{8 h^{-1}}$ to decrease (increase) with decreasing $\Omega_{0}$. In Figure 3 we show two $\Lambda=0$ open models, in which $\Omega_{0}=0.35$ and 0.2 (lines labeled B1 and $\mathrm{B} 2$, respectively). The values of $\sigma_{8 h^{-1}}$ and $n$ in these models, which are consistent with both the $C O B E$ and cluster normalizations, are $(0.87,1.15)$ and $(0.87,1.2)$, respectively. Note that the values of $t_{0}$ and $\epsilon_{Q}$ also need to be readjusted in these cosmologies. We find $\left(t_{0}, \epsilon_{Q}\right)=\left(10^{6.2} \mathrm{yr}, 10^{-3.7}\right)$ for $\Omega_{0}=0.35$ and $\left(t_{0}, \epsilon_{Q}\right)=\left(10^{6.8} \mathrm{yr}, 10^{-3.9}\right)$ for $\Omega_{0}=0.2$. The lines in the bottom panel labeled B1 and B2 show that the $z \sim 3$ number counts indeed flattens at $V \sim 23$ and $V \sim 24$ in these models. The upper panel of this figure also shows that in the $\Omega_{0}=0.35$ model the cumulative $z>3.5$ counts at $V=29$ are lowered to the required level of $\sim 2$ objects. While the $z>3.5$ counts in the $\Omega_{0}=0.2$ model are also reduced, the predicted number of miniquasars at the HDF limit is still too large in this case (i.e., $\sim 3$ objects, making the observed lack of detections a $3 \sigma$ event).

A third alternative approach to the photoionization feedback for reducing the faint $z>3.5$ counts is to make the LF decline faster at high redshifts. While the quasar LF is reasonably well determined at $z \sim 3$, it is significantly more uncertain around $z \sim 4$ (see Figs. 1 and 2 in Pei 1995). One could exploit this uncertainty and modify the models so that the predicted number of $z \sim 4$ objects is at the low end of the allowed range. We have found that the redshift evolution cannot be made sufficiently steep by changing the cosmology or the power spectrum while still maintaining a good fit to the LF around $z \sim 3$. However, the evolution can be made steeper if the halo mass associated with a fixed luminosity is increased, since the formation rate of higher mass halos evolves more rapidly. To achieve this change, we introduce a simple dependence of luminosity on halo mass, $L=\epsilon_{0}\left(M_{\text {halo }} / 10^{8} M_{\odot}\right)^{1+\alpha}$, with $\alpha>0$. A similar scaling, including an additional redshift dependence, $L \propto M_{\mathrm{bh}} \propto$ $v_{\text {circ }}^{5} \propto M_{\text {halo }}^{5 / 3}(1+z)^{5 / 2}$, has been recently considered by Haehnelt et al. (1998). Our adopted scaling lacks the factor $(1+z)^{5 / 2}$ and therefore predicts a steeper redshift evolution and a slightly smaller number of $z>3.5$ objects than in Haehnelt et al. (1998). Since most objects at $V=29$ are located at $z<6$, the difference in the number counts predicted by the two models in the relevant magnitude range is small ( $\lesssim 15 \%)$.

For any given value of $\alpha$, the fitting procedures described in $\S 4$ can be repeated to obtain the values of $\epsilon_{0}$ and $t_{0}$. Although by definition our procedure for quasars fits the $\mathrm{LF}$ at $2.6<z<4$, an increase in the value of $\alpha$ yields an increase in the characteristic mass of the LF and a decrease in the total number of faint $z>3.5$ miniquasars. We have experimented with values of $0<\alpha<1$ and found that for $\alpha \gtrsim 0.5$ the fitting procedure for quasars fails because of the implausibly steep redshift evolution of the model LF already for the observed redshift interval $2.6<z<4$. In other words, an acceptable fit in our models to the LF at $z=3$ would result in a predicted $z \sim 4 \mathrm{LF}$ that falls below the error bars of the observed $z=4$ bin in Pei (1995). Note that much stronger deviations from the linear relation $M_{\mathrm{bh}} \propto M_{\text {halo }}$ would also be mildly inconsistent with observational data for local galaxies (Magorrian et al. 1998; Kormendy et al. 1997; McLeod 1997). The largest plausible reduction in the faint counts can therefore be achieved using $\alpha=0.5$. In this case, we obtain $M_{\mathrm{bh}} / M_{\text {halo }}=$ $8 \times 10^{-7}\left(M_{\text {halo }} / 10^{8} M_{\odot}\right)^{0.5}$ and $f(t)=\exp \left(-t / 3 \times 10^{6} \mathrm{yr}\right)$ and predict the number counts shown by the curves labeled $\mathrm{C}$ in Figure 3. The bottom panel shows that the quality of the fit to the $z \sim 3 \mathrm{LF}$ at $17<V<22.5$ is comparable with the fit in our standard model. The top panel shows that relative to model A, the $z>3.5$ counts decrease by a factor of 3 to $\sim 3$ objects. However, even with the largest allowed value of $\alpha$, the $z>3.5$ counts remain a too high (i.e., $\sim 3$ objects, making the HDF a $3 \sigma$ event).

A fourth effect that could contribute to the deficit of observed faint miniquasars is the expected shift in the "big blue bump" (BBB) component of the quasar spectrum to 
higher energies for the lower mass black holes that populate the universe at high redshifts. In thin accretion disk models (e.g., Peterson 1997) the emission temperature, and hence the peak wavelength of the BBB, scales with the black hole mass as $\lambda_{\text {peak }} \propto M_{\mathrm{bh}}^{0.25}$. In our present template spectrum, which is based on data from local $(z \lesssim 1)$ quasars (Elvis et al. 1994), the BBB peak is tentatively located at $1000 \AA$ (see HL98). Since the characteristic black hole might be smaller by several orders of magnitude at $z \sim 4-6$ than at $z \lesssim 1$, the BBB feature might shift by a factor of a few in wavelength. Such a shift would reduce the observed $V$ band flux by a factor of $\sim 7$ and the rest frame $B$-band flux (which we use to calibrate our model) by a factor of $\sim 2$. In particular, if the $\mathrm{BBB}$ in high-redshift miniquasars is blueshifted to $\lesssim 300$ $\AA$, then the faint $V$ counts would be reduced by a factor of $\sim 2$, which is not quite sufficient to bring the number counts into agreement with the HDF data.

Finally, we mention the possibility that the host galaxies of miniquasars may actually be resolved by the HST. Indeed, another way to explain the discrepancy between models and observations is to consider host galaxies that are sufficiently bright to outshine their miniquasars. Such hosts, if resolved, would prevent their miniquasars from being detected and classified as point sources. Note, however, that although miniquasars are rather faint, in our models their flux is still $\sim 100$ times higher than expected from a dwarf galaxy with the same halo mass (see $\S 7$ below).

\section{NUMBER COUNTS OF $z>3.5$ GALAXIES}

The predicted number of faint $z>3.5$ galaxies in our models at the HDF limits is a factor of 5-35 higher than the number of miniquasars. However, faint galaxies might have sufficiently large angular sizes so as to appear extended and avoid the overprediction of point sources.

A simple estimate shows that this might indeed be the case (see also Haiman \& Loeb 1998a). To estimate what fraction of the $z>3.5$ galaxies may have angular diameters above the 0.1 pixel size of the three wide field cameras (WFC), we assume that each collapsed halo has a $1 / r^{2}$ density profile, with an overdensity of 50 at the edge of the halo relative to the average background density (equivalent to an average overdensity of 150) and that the luminous region of the galaxy extends out to $5 \%$ of the virial radius of the halo (see Navarro, Frenk, \& White 1995). With these assumptions, we find that for the low star formation efficiency (producing an average metallicity of $Z_{\mathrm{Ly} \alpha}=10^{-3} Z_{\odot}$ in the intergalactic medium), only $0.5 \%$ of the $z>3.5$ objects would be unresolved at $V=29.8$. As a result, the $\mathrm{HDF}$ is expected to contain no pointlike galaxies at this magnitude limit. The unresolved fractions around the HDF sensitivity limit are significantly higher for a high star formation efficiency $\left(Z_{\mathrm{Ly} \alpha}=10^{-2} Z_{\odot}\right)$. In this case, a fraction equal to $2 \%, 9 \%, 18 \%$, and $24 \%$ of the $z>3.5$ objects would be unresolved at $V=27.5,28.0,28.9$, and 29.8, respectively. Therefore, given the expected intrinsic sizes of the galaxies, the overprediction problem disappears at low star formation efficiencies but is not fully resolved if the star formation efficiency is higher and the apparent angular size of galaxies smaller. This could be interpreted as support for a low star formation efficiency and a corresponding low average metallicity of the IGM at $z \sim 3$.

It is interesting to examine the sensitivity of the number counts to the underlying cosmology and to the relation between $M_{\text {star }}$ and $M_{\text {halo }}$, assuming that the stars occupy a region much smaller than $5 \%$ of the virial radius of each halo, so that most $z>3.5$ galaxies are unresolved. Obviously, it is more difficult to reconcile the number counts of such galaxies with the HDF data than that of quasars. There are two reasons for this result. First, the number counts of galaxies are higher to begin with. Second, the model galaxies are $\sim 2$ orders of magnitude less luminous than quasars, so the same $V$ magnitude corresponds to a halo mass that is larger by $\sim 2$ orders of magnitude. The differential number counts flatten at the fixed mass $M_{*}$; for galaxies this mass corresponds to $V \sim 30$ at $z=4.5$.

As an example, the curve labeled B in Figure 4 shows the galaxy counts in the $\Omega_{0}=0.35$ open CDM model. The counts are somewhat less steep than in the $\Lambda$ model (the solid curve labeled A). Although the flattening of the mass function at $M<M_{*}$ reduces the faint counts, the effect is negligible at $V \sim 29$. The figure also shows the results of changing the dependence of the star formation efficiency on the halo mass (changing $\alpha$ ). When the star formation efficiency is normalized to $Z_{\mathrm{Ly} \alpha}=10^{-3} Z_{\odot}$, then $\alpha=2$ and $\epsilon_{0}=3.3 \times 10^{-9}$ are required for consistency with no detections (see the line labeled C2). Similarly, when $Z_{\mathrm{Ly} \alpha}=10^{-2}$ $Z_{\odot}$ is assumed, $\alpha=2.5$ and $\epsilon_{0}=7.3 \times 10^{-12}$ are required (C1). As the figure shows, the slopes of the latter number count curves are flattened considerably. For reference, we also show the $V$-band number counts of all extended sources observed in the HDF. Only a small fraction of all detected objects at $V=29$ are expected in our models to have $z>3.5$; most of the faint sources are actually at lower redshifts. Finally, we note that an additional reduction of the galaxy counts could also be caused by dust extinction. Estimates based on the Calzetti, Kinney, \& StorchiBergmann (1994) extinction curves indicate that the number of $z \lesssim 3$ sources could be as much as $\sim 7$ times higher than currently detected (Pettini et al. 1997) when dust reddening is taken into account.

Given all of the above uncertainties, we conclude that, unlike in the case of miniquasars, there is no significant discrepancy between the expected galaxy counts and the lack of faint point sources. This is true as long as the stars occupy a region extending out to a few percent of the virial radius of their host halo and the star formation efficiency is close to the value expected from a low average $\operatorname{Ly} \alpha$ forest metallicity, $Z_{\mathrm{Ly} \alpha}=10^{-3} Z_{\odot}$.

\section{CONCLUSIONS}

The observed lack of faint, unresolved, and red sources in the HDF down to $V \approx 29$ requires models that are consistent with the data at the $2 \sigma$ level to predict no more than two pointlike high- $z$ objects to this limiting magnitude. Given that the observations are $\sim 50 \%$ complete, this appears to be marginally consistent with the predicted number $(\sim 4)$ of high-redshift miniquasars in the simplest hierarchical models, which suppress the formation of black holes in CDM halos with circular velocities, $v_{\text {circ }} \leq 50 \mathrm{~km}$ $\mathrm{s}^{-1}$. Without a photoionization feedback, these models predict $\sim 8$ miniquasars from $z>3.5$. In models without UV feedback, the predicted miniquasar counts might be reduced by early structure formation (so that $M_{*} \gtrsim 10^{11}$ $M_{\odot}$ by $z=3$ ), but only if one postulates that new black holes do not form in halos below the nonlinear mass scale, $M_{*}(z)$. An example for such a model is the $\Omega_{0}=0.35$ open CDM model, with a power spectrum slope of $n=1.15$ and normalization of $\sigma_{8 h^{-1}}=0.87$. The $z>3.5$ number counts 


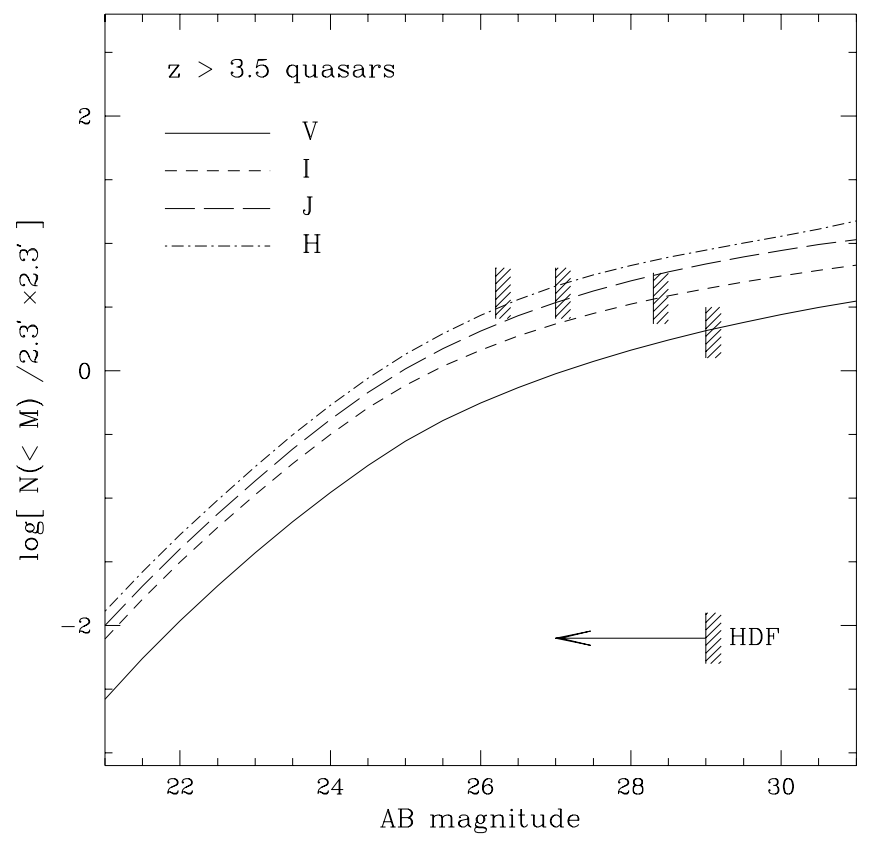

FIG. 5.- $V, I, J$, and $H$ counts for quasars in the $\Lambda$ CDM model with $\Omega_{0}=0.35$ and minimum circular velocity $v_{\text {circ }}=75 \mathrm{~km} \mathrm{~s}^{-1}$. This model is consistent with HDF data in the $V$ band at the $5 \%$ level. Sensitivities shown for each band are for a fixed signal-to-noise ratio and exposure time.

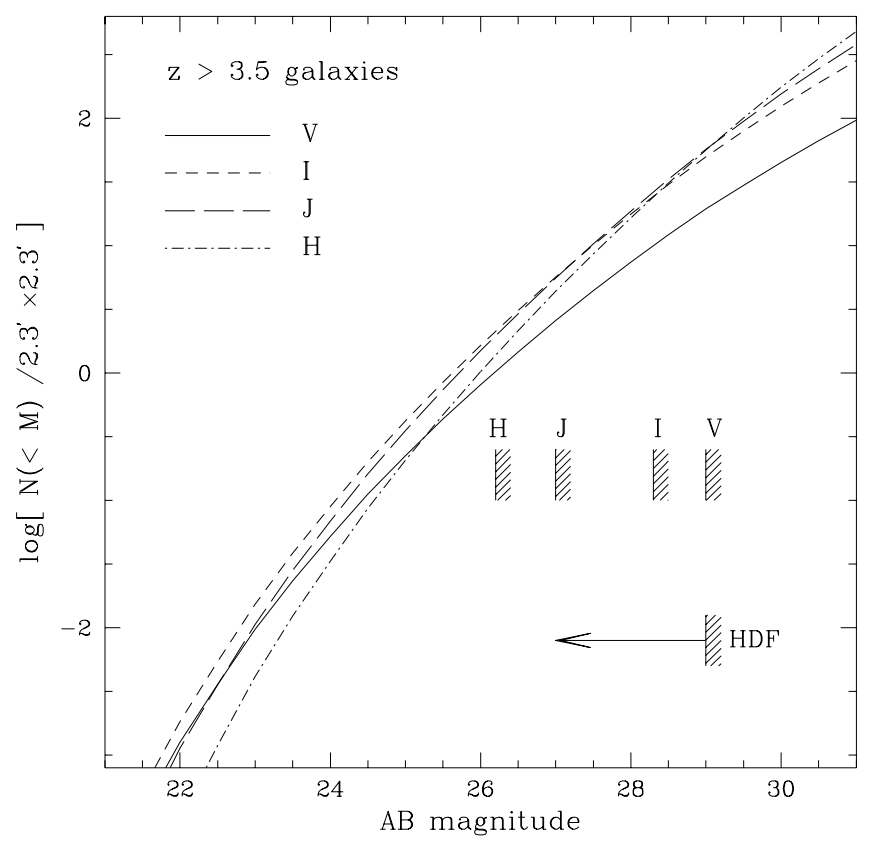

Fig. 6.-Theoretical $V, I, J$, and $H$ counts for $z>3.5$ galaxies in our standard $\Lambda$ CDM model with $Z_{\mathrm{Ly \alpha}}=10^{-3} Z_{\odot}$. Since most galaxies at $V<30$ mag may be resolved, this model could still be consistent with the HDF images. can be further reduced by introducing a black hole formation efficiency, $M_{\mathrm{bh}} / M_{\text {halo }}$, that depends on the halo mass as $\propto M_{\text {halo }}^{0.5}$. Similarly, a shift of the BBB component to shorter wavelengths in the spectra of faint miniquasars could also help reduce the predicted counts. Combined with a minimum circular velocity of $v_{\text {circ }}=50 \mathrm{~km} \mathrm{~s}^{-1}$, either of these latter two effects could reduce the number counts to less than $\sim 2$ objects. On the other hand, we find that without a photoionization feedback neither of these effects would be sufficient by itself to explain the lack of detections.

The models predict $\sim 5-35$ times more $z>3.5$ galaxies than miniquasars at $V=29$. If the star formation rates in these galaxies are adjusted to produce an average Ly $\alpha$ forest metallicity of $10^{-2} Z_{\odot}$ by $z=3$, then more than $10 \%$ of all galaxies could appear unresolved at the 0.1 pixel size of the WFC, possibly leading to another discrepancy with the observed lack of detections. The situation changes if the star formation rate is lowered by an order of magnitude (normalized to produce a $10^{-3} Z_{\odot}$ average IGM metallicity at $z \sim 3$ ), since then almost all of the predicted $z>3.5$ galaxies are expected to be extended rather than point sources.

Future observations at longer IR wavelengths, such as the $J$ and $H$ infrared bands, would be better suited to the study of the $z \gtrsim 4.5$ universe, as they allow bigger volumes to be probed. In Figure 5 we show the expected number counts of miniquasars in the $V, I, J$, and $H$ bands for the model, which is consistent with the optical HDF data. An increasingly larger number of objects are predicted to be detected in the $I, J$, and $H$ bands, as these are affected by intervening absorption only at extreme redshifts. These number counts could be somewhat reduced, since faint, compact WFC sources lose the signal-to-noise ratio when observed by NICMOS because of the poorer angular resolution of the IR images. Figure 6 shows the expected number of galaxies in the infrared bands. At $30 \mathrm{mag}$, the gain in observing galaxies redward of the $I$ band is not as significant as for miniquasars, because the intrinsic flux of unreddened galaxies, unlike those of quasars, decreases as a function of increasing wavelength at $z \sim 4$. The redshifts of either type of sources can be obtained using a straightforward analogy to the $B$ dropout technique applied to the $I-J$ versus $J-H$ color-color diagram shown in Figure 2. Future deep observations with NICMOS in these bands of a field comparable in size with the HDF would be able to either reveal tens of ultra-high redshift miniquasars or else place tighter constraints on quasar models than currently possible using $V$ and $I$ data.

We thank Steve Beckwith for discussions and Martin Haehnelt and Martin Rees for useful comments. Z. H. thanks G. Djorgovski for suggesting to predict number counts of high-redshift sources in different bands. P. M. acknowledges support by NASA through grants NAG54236 and AR-06337.10-94A from the Space Telescope Science Institute. A. L. acknowledges support from NASA ATP grant NAG5-3085 and the Harvard Milton fund.

\section{REFERENCES}

Babul, A., \& Rees, M. J. 1992, MNRAS, 255, 346

Bajtlik, S., Duncan, R. C., \& Ostriker, J. P. 1988, ApJ, 327, 570

Baugh, C. M., Cole, S., Frenk, C. S., \& Lacey, C. G. 1998, ApJ, 498, 504 Bruzual, G., \& Charlot, S. 1996, available from gemini.tuc.noao.edu Calzetti, D., Kinney, A. L., \& Storchi-Bergmann, T. 1994, ApJ, 429, 582 Carroll, S. M., Press, W. H., \& Turner, E. L. 1992, ARA\&A, 30, 499
Cole, S., Aragón-Salamanca, A., Frenk, C., Navarro, J., \& Zepf, S. 1994, MNRAS, 271, 781

Conti, A., Kennefick, J. D., Martini, P., \& Osmer, P. S. 1998, preprint (astro-ph/9808020)

Efstathiou, G. 1992, MNRAS, 256, 43

Efstathiou, G., \& Rees, M. J. 1988, MNRAS, 230, 5p 
Eisenstein, D. J. 1997, preprint (astro-ph/9709054)

Eisenstein, D. J., \& Loeb, A. 1995, ApJ, 443, 11

Elson, R. A. W., Santiago, B. X., \& Gilmore, G. F. 1996, NewA, 1, 1

Elvis, M., Wilkes, B. J., McDowell, J. C., Green, R. F., Bechtold, J., Willner, S. P., Oey, M. S., Polomski, E., \& Cutri, R. 1994, ApJS, 95, 1

Flynn, C., Gould, A., \& Bahcall, J. N. 1996, ApJ, 466, L55

Gnedin, N. Y., \& Ostriker, J. P. 1997, ApJ, 486, 581

Governato, F., Baugh, C. M., Frenk, C. S., Cole, S., Lacey, C. G., Quinn, T., \& Stadel, J. 1998, Nature, 392, 359

Haehnelt, M. G., Natarajan, P., \& Rees, M. J. 1998, preprint (astro-ph/ 9712259)

Haehnelt, M. G., \& Rees, M. J. 1993, MNRAS, 263, 168

Haiman, Z., \& Loeb, A. 1997, ApJ, 483, 21 (HL97) . 1998a, in ASP Conf. Ser. 133, Science with the Next Generation Space Telescope, ed. E. Smith \& A. Koratkar (San Francisco: ASP), 251 1998b, ApJ, 503, 505 (HL98)

Haiman, Z., Rees, M. J., \& Loeb, A. 1997, ApJ, 476, 458

Ikeuchi, S. 1986, Ap\&SS, 118, 509

Kauffmann, G., Guiderdoni, B., \& White, S. D. M. 1994, MNRAS, 267, 981

Kauffmann, G., \& White, S. D. M. 1993, MNRAS, 261, 921

Kormendy, J., et al. 1997, ApJ, 482, L139

Lacey, C., \& Cole, S. 1993, MNRAS, 262, 627

Loeb, A. 1998, in ASP Conf. Ser. 133 Science with the Next Generation Space Telescope, ed. E. Smith \& A. Koratkar (San Francisco: ASP), 73

Madau, P. 1995, ApJ, 441, 18

Madau, P., Ferguson, H. C., Dickinson, M. E., Giavalisco, M., Steidel, C. C., \& Fruchter, A. 1996, MNRAS, 283, 1388

Magorrian, J., et al. 1998, AJ, 115, 2285

McLeod, K. 1997, in Proc. ESO-IAC Conf., ed D. L. Clements \& I. PérezFournon (Berlin: Springer), 45

Méndez, R. A., Minniti, D., De Marchi, G., Baker, A., \& Couch, W. J. 1996, MNRAS, 283, 666
Navarro, J. F., Frenk, C. S., \& White, S. D. M. 1995, MNRAS, 275, 56

Navarro, J. F., \& Steinmetz, M. 1997, ApJ, 478, 13

Ostriker, J. P., \& Steinhardt, P. J. 1995, Nature, 377, 600

Pei, Y. C. 1995, ApJ, 438, 623

Peterson, B. M. 1997, An Introduction to Active Galactic Nuclei (Cambridge: Cambridge Univ.)

Pettini, M., Steidel, C. C., Adelberger, K. L., Kellogg, M., Dickinson, M. E., \& Giavalisco, M. 1997, in ASP Conf. Ser. 148, Origins, ed. J. M. Shull, C. E. Woodward, \& H. Thronson (San Francisco: ASP), in press

Press, W. H., \& Schechter, P. L. 1974, ApJ, 181, 425

Quinn, T., Katz, N., \& Efstathiou, G. 1996, MNRAS, 278, 49

Scalo, J. M. 1986, Fundamentals of Cosmic Physics, vol. 11, p. 1-278

Schaller, G., Schaerer, D., Meynet, G., \& Maeder, A. 1992, A\&AS, 96, 269

Small, T. A., \& Blandford, R. D. 1992, MNRAS, 259, 725

Smith, E. P., \& Koratkar, A. (ed.) 1998, ASP Conf. Ser. 133, Science with the Next Generation Space Telescope

Somerville, R. S., \& Primack, J. R. 1998, in preparation

Songaila, A. 1997, ApJ, 490, L1

Songaila, A., \& Cowie, L. L. 1996, AJ, 112, 335

Steidel, C. C., Adelberger, K. L., Dickinson, M. E., Giavalisco, M., Pettini, M., \& Kellogg, M. 1998, ApJ, 492, 428

Thoul, A. A., \& Weinberg, D. H. 1996, ApJ, 465, 608

Tytler, D., et al. 1995, in QSO Absorption Lines, ESO Astrophys. Symp., ed. G. Meylan (Heidelberg: Springer), 289

Viana, P. T. P., \& Liddle, A. R. 1996, MNRAS, 281, 323

Vogeley, M. S. 1998, ApJ, in press

Williams, R. E., et al. 1996, AJ, 112, 1335

Zheng, W., Kriss, G. A., Telfer, R. C., Grimes, J. P., \& Davidsen, A. F. 1997, ApJ, 475, 469 